\title{
Theoretical Studies of Comets in the 55 Cancri System
}

\author{
Rudolf Dvorak ${ }^{1}$, Birgit Loibnegger ${ }^{1}$ and Manfred Cuntz ${ }^{2 \star}$ \\ ${ }^{1}$ Institute of Astrophysics, University of Vienna, Türkenschanzstraße 17, A-1180 Vienna, Austria \\ ${ }^{2}$ Department of Physics, University of Texas at Arlington, Arlington, TX 76019, USA
}

Accepted XXX. Received YYY; in original form ZZZ

\begin{abstract}
We present orbital integrations for various Jupiter family comets (JFCs) in the 55 Cancri system. This star is known to possess (at least) five planets with masses ranging from super-Earth to Jupiter-type. Furthermore, according to observational constraints, there is a space without planets between $\sim 0.8$ au and $\sim 5.7 \mathrm{au}$, offering the principal possibility of habitable terrestrial planets, including long-term orbital stability. Hence, there is a stark motivation for the study of comets in the 55 Cnc system noting that comets are viewed a viable candidate mechanism for the delivery of water to Earthtype planets located in stellar habitable zones. However, our study shows that the duration of stability of JFC analogs in the 55 Cnc system is much shorter compared to comets in the Solar System owing to profound differences in the planetary structure of the systems. For example, between planet 55 Cnc-f and 55 Cnc-d, the comets do not undergo close cometary encounters akin to Earth and Mars in the Solar System as the planetary masses in the 55 Cnc system are much larger than in the Solar System and therefore the comets are much less orbitally stable. Nevertheless, we expect an increased number of comet-planet encounters as well as cometary collisions in the $0.8 / 5.7$ au gap. Future observations and additional theoretical studies are required to shed light on the possibility of habitable terrestrial planets in the 55 Cnc system, including the possible role(s) of exocomets in the facilitation of planetary habitability.
\end{abstract}

Key words: astrobiology - circumstellar matter - comets: general - methods: numerical - protoplanetary disks - stars: individual (55 Cnc)

\section{INTRODUCTION}

An active field of research is the study of comets around stars other than the Sun, also referred to as exocomets. Previous observational results have been given by, e.g., Beust et al. (1990), Kiefer et al. (2014), Eiroa et al. (2016), and Rappaport et al. (2018). Important topics of interest relevant to the fields of astrophysics and astrobiology include (1) studies on the origin of comets in different types of systems, (2) gravitational interaction of comets with respect to stars and their planetary systems and (3) the proliferation of water and possible biomolecules to Earth-type planets, including planets situated in the stellar habitable zones; see, e.g., Stern (1987) and Raymond \& Izidoro (2017) for background information. Recently, the research group centered at Vienna, Austria, made various contributions to that field, including model simulations for the systems of HD 10180, Proxima Centauri, 47 UMa, and HD 141399; see Loibnegger et al. (2017), Schwarz et al. (2018), Cuntz et al. (2018), and Dvorak et al. (2020), respectively.

Here we investigate the extrasolar planetary system

^ E-mail: cuntz@uta.edu (MC)
55 Cnc with a particular focus on (analogs of) Jupiter family comets (JFC) found in our Solar System. Previous studies on the origin and dynamics of Solar System comets have been given by, e.g., Levison \& Duncan (1997), Emel'yanenko et al. (2013), and Fouchard et al. (2017, 2018). An important motivation for our efforts stems from the fundamentally different structures of the two systems as identified by the different masses and star-planet distances of the system planets. Although 55 Cancri is a stellar binary with its components readily referred to as 55 Cnc A and 55 Cnc B, we do not consider the companion $55 \mathrm{Cnc} \mathrm{B}$ in our integrations owing to its small mass ( $\sim 25 M_{\odot}$; see Gaidos et al. 2014 and Newton et al. 2017) and the large separation distance of the two stellar components given as about 1000 au (Duquennoy \& Mayor 1991). In fact, the influence of 55 Cnc B (and of any planet hosted by that component) on the cometary orbits associated with the star-planet system of $55 \mathrm{Cnc} \mathrm{A}$ is negligible.

Further simplifications are discussed below in more detail. Specifically, in our numerical simulations we only include the planets 55 Cnc-c, 55 Cnc-f, and 55 Cnc-d (see Table 1). The other two planets, i.e., 55 Cnc-b and 55 Cnce, orbit the star in relatively close proximity $(d<0.12 \mathrm{au})$, 
which makes cometary encounters with these planets highly improbable. This view receives support from checking the respective perihelion distances in the list of all JFCs (i.e., $\omega<0.336$ au except for three $\mathrm{SOHO}^{1}$ comets, which are $321 \mathrm{P}, 322 \mathrm{P}$, and $323 \mathrm{P}$ with $\omega \approx 0.05 \mathrm{au}$, with $\omega$ being the pericentric distance). Moreover, most of the JFCs have their closest encounters to the Sun outside Earth's orbit.

In this work, we focus on 55 Cancri ( $55 \mathrm{Cnc}, \rho^{1} \mathrm{Cnc}$ ), a G8 V star (Gonzalez 1998), with an effective temperature $^{2}$ and luminosity lower than those of the Sun (e.g., Fischer \& Valenti 2005; Ligi et al. 2016). Note that $55 \mathrm{Cnc}$ is considerably older than the Sun with an estimated age of 8 Gyr (Mamajek \& Hillenbrand 2008). Moreover, $55 \mathrm{Cnc}$ has a mass of $0.96 M_{\odot}$ (Ligi et al. 2016); it is therefore considered an orange dwarf. Additionally, 55 Cnc is known to host five planets, discovered between 2008 and 2011 by Fischer et al. (2008), Dawson et al. (2008), and Winn et al. (2011) based on the radial velocity method. All of these planets have masses significantly larger than Earth. However, based on previous theoretical studies, see, e.g., von Bloh et al. (2003), Rivera \& Haghighipour (2007), Raymond et al. (2008), Smith \& Lissauer (2009), and Satyal \& Cuntz (2019), the existence of Earth-mass planets in that system is highly plausible.

Studies of $55 \mathrm{Cnc}$, as well as of similar stars, are of particular interest to astrobiology in consideration of the various favorable properties of general orange dwarfs, i.e., late-type $\mathrm{G}$ and early-type $\mathrm{K}$ main-sequence stars (e.g., Cuntz \& Guinan 2016; Lingam \& Loeb 2018, 2019; Schwieterman et al. 2019). These stars are considered particularly suitable for hosting planets with exolife in light of the numerous features supportive of exobiology, including (but not limited to) the frequency of those stars, the relatively large size of their HZs (if compared to M dwarfs), and their relatively long main-sequence life times (i.e., 15 Gyr to 30 Gyr, compared to 10 Gyr for solar-like stars). A detailed analysis of the physical constraints on the likelihood of life on exoplanets in various stellar environments has been given by Lingam \& Loeb (2018). Note that 55 Cnc is expected to possess many of those features.

Our paper is structured as follows: In Section 2, we present our methods and the numerical setup. In Section 3, we convey our results and discussion, including studies of the (hypothetical) 'Jupiter family' in $55 \mathrm{Cnc}$ as well as simulations for comet analogs of the Solar System comets 2P / Encke, 9P / Tempel 1, and 31P / SchwassmannWachmann 2. Our conclusions and comments regarding possible future research are given in Section 4.

\section{METHODS AND NUMERICAL SETUP}

For our study we assume that 55 Cancri harbors an Oorttype cloud of comets at the system's outskirts. These comets

\footnotetext{
1 The SOlar and Heliospheric Observatory (SOHO) studies the internal structure of the Sun as well as its extended outer atmosphere including the origin of the solar wind.

${ }^{2}$ Further determinations of stellar parameters for $55 \mathrm{Cnc}$ have been given by von Braun et al. (2011); they are consistent with those adopted in this study.
}

are expected to be gravitationally disturbed by, e.g., passing stars or through galactic tidal forces, resulting in highly eccentric cometary orbits, thus allowing the comets to enter the system's inner domain. Cometary trajectories, including their long-term developments, as well as mechanisms of cometary injections into the system have previously been studied by numerous authors (e.g., Fouchard et al. 2017; Rickman et al. 2017; Dvorak et al. 2020, and references therein).

The goal of the current study is to explore the dynamics of comets, including their interaction with the planets of the 55 Cancri system. We will examine the dynamics of some Solar System comet analogs ${ }^{3}$, namely, 2P / Encke, 9P / Tempel 1, and 31P / Schwassmann-Wachmann 2; they are later referred to as comet A, B, and C, respectively, for convenience. We will also include a fictitious Earth-mass planet in order to investigate its influence on the trajectories of the comets. This planet is put in a stable orbit with a semimajor axis of $1 \mathrm{au}$.

For information on the 55 Cancri system including its five planets, see Table 1; However, contrary to the recent study by Satyal \& Cuntz (2019), we include the two inner planets in a limited sense only by adding their masses to the mass of the central star. This is a common practice routinely also adopted for the integration of the Solar System. In this case, Mercury's mass is added to the Sun's mass when the desired precision is not at the limit of the integration itself (1.0E-15 for double precision computations). However, regardless of the process, the various planetary parameters for the 55 Cnc system, used as initial conditions, are not very well constrained, especially the planetary masses are in fact minimum masses due to their derivation from radial velocities observations.

Our method is based on a direct numerical integration with Lie-series of the equations of motion, a method readily used for computations for $N$-body integrations - it has already been compared to other methods for numerical integration in various papers (e.g., Hanslmeier \& Dvorak 1984). This method solves the differential equations by differentiation that leads to a power series with factors computed in advance. This approach is quite fast and competitive compared to those used by many other authors. In addition, the length of the time-step can be changed separately for every step; this approach increases the precision of the method, which is particularly helpful for close encounters and collisions (e.g., Dvorak et al. 2020). Based on the data of Table 1, the two innermost planets 55 Cnc-e and 55 Cnc-b with orbital periods of less than a day and $\sim 15$ days, respectively, would entail very small step sizes for the integration. Therefore, their masses have been added to the mass of the central star.

\footnotetext{
3 Solar comets put into the 55 Cnc system are referred to as comet analogs; their initial values, except for their semimajor axes, have been chosen to match those of the respective solar system comet. Comet clones denote sets of comets created through variations of their $M A$ values, which is done for the purpose of detailed numerical simulations.
} 
Table 1. Orbital Parameters of the 55 Cancri Planets

\begin{tabular}{lccccccc}
\hline $\begin{array}{l}\text { Planet } \\
\ldots\end{array}$ & $\begin{array}{c}\text { Mass } \\
\left(M_{\oplus}\right)\end{array}$ & $\begin{array}{c}a \\
(\mathrm{au})\end{array}$ & $\begin{array}{c}\boldsymbol{N} \\
\ldots\end{array}$ & $\begin{array}{c}i \\
\left(^{\circ}\right)\end{array}$ & $\begin{array}{c}\omega \\
\left(^{\circ}\right)\end{array}$ & $\begin{array}{c}\text { MA } \\
\left(^{\circ}\right)\end{array}$ & $\begin{array}{c}\text { Orbital Period } \\
(\text { days })\end{array}$ \\
\hline 55 Cancri e & 8.37 & 0.0156 & 0.170 & 7.5 & 90 & 204.032 & 0.7365 \\
55 Cancri b & 264.75 & 0.1148 & 0.010 & 0.0 & 110 & 19.88 & 14.650 \\
55 Cancri c & 54.38 & 0.2403 & 0.005 & 0.0 & 356 & 116.10 & 44.364 \\
55 Cancri f & 57.209 & 0.781 & 0.320 & 0.0 & 139 & 198.61 & 259.80 \\
55 Cancri d & 1169.6 & 5.74 & 0.020 & 0.0 & 254 & 10.89 & 5169.0 \\
"Earth" & 1.0 & 1.0 & 0.0 & 0.0 & 0.0 & 0.0 & $\ldots$ \\
\hline
\end{tabular}

Note: The planetary parameters (mass, $a, e, i, \omega$, and $M A$, with all parameters having their usual meaning) of the 55 Cancri planets are listed, see references Fischer et al. (2008), Dawson et al. (2008), Winn et al. (2011), Endl et al. (2012), and Ligi et al. (2016)). The ascending node $\Omega$ is set to zero for all planets.

\section{RESULTS AND DISCUSSION}

\subsection{The 'Jupiter family' in 55 Cancri}

The 580 JFC examined here have been placed into the 55 Cnc system with their orbital elements adopted from the JPL Small-Body Database ${ }^{4}$ The focus of our work is to study various Solar System comet system analogs in further detail. However, the values for their semimajor axes have been normalized based on Jupiter's semimajor axis and that of 55 Cnc-d. The other elements, i.e., the cometary eccentricities and inclinations (see Table 2) were left unchanged. For each comet analog we created 100 initial conditions based on slight variations in their initial mean anomalies $M A$, bracketed by $M A=0^{\circ}$ and $M A=360^{\circ}$ with a step size of $\delta M A=3.6^{\circ}$, which led to a total of almost 60,000 integrated orbits. The total integration time for each orbit was set to 100 kyr.

The results for each comet were analyzed with respect to their mean escape times and the number of remaining comets, see Figures 1 to 4 . In the following, we explain the plots while focusing on the results for three examples chosen in regard to a large, medium, and small cometary eccentricity. In order to further compare the dynamical evolution for the three comets placed in the 55 Cnc system relative to the Solar System we adopted two models: one that includes the planets Venus to Saturn (Ve2Sa) and another one excluding Saturn (Ve2Ju); see Table 3.

The outcome is that the lifetimes of the comets in 55 Cnc are significantly shorter compared to the corresponding comets in the Solar System because of the large masses of 55 Cnc-f and 55 Cnc-d. This is especially true for comet A owing to the large eccentricities of the respective comet clones; their close encounters to the 55 Cnc system planets expel most of them from the system as only 3 out of 100 of the comet clones are able to survive. This result is drastically different for simulations of the Solar System where only a few of the comet A clones were ejected (with an ejection defined as $e \geq 1$ ); i.e., 2 and 1 for the Ve2Sa model and the Ve2Ju model, respectively. After a total integration time of 100 kyr, only 42 of the 100 comet clones for 55 Cnc survived

${ }^{4}$ For information see link: ssd.jpl.nasa.gov/sbdb.cgi. for comet B, and only 2 of the clones for comet C. The reason for the few remaining clones for comets $\mathrm{A}$ and $\mathrm{C}$ is their close proximity to $55 \mathrm{Cnc}$-d, which in combination with the cometary eccentricities result in close planetary encounters shortly after start of the integrations; therefore, many of the comet clones are ejected soon after. Hence, it is found that the JFCs within the Solar System are much more stable compared to the corresponding comets hosted by 55 Cnc.

\subsection{Lifetime of Comets}

\subsubsection{General Aspects}

Figures 1 - 4 depict the mean escape times (in red) and the mean numbers of the remaining comet clones (out of 100; in blue) for various case studies. We omit showing the figures for the faint comets no. 351 to 580 , which are not significantly different from the previous ones. Some comets do not escape during the integration time of $100 \mathrm{kyr}$, which is attributable to the fact that these orbits are stable for thousands of orbital periods. Note that in some cases the red and blue filled circles overlap (e.g., comet 151 in the left panel of Figure 3). In this particular case, the escape time has been equal to the integration time for all 100 clones of this particular comet. This means that all 100 clones are stable for the entire time of integration.

On the other hand, in some cases there are no surviving comet clones in our simulations. These kinds of orbits are highly unstable due to their very large eccentricities of $e>$ 0.97; in case of the Sun, those types of comets have been discovered by SOHO. In conclusion, we find that most of the $\sim 60,000$ comets have a mean escape time below $20 \mathrm{kyr}$ and the mean number of surviving comets remains in most cases below 40 of 100 clones. Table 4 lists the stable JFCs analogs for the 55 Cnc system based on simulations extending to 100 kyr.

\subsubsection{The Comet 2P / Encke}

Figure 5 depicts the orbits of the clones for comet A at the beginning and end of the integration. One can see that after a short time, i.e., about $200 \mathrm{yr}$, the comet clones start experiencing the perturbations by the planets; consequently, their semimajor axes increase leading to possible escapes from the 
Table 2. Orbital Information for Solar System Comet Analogs

\begin{tabular}{llcccc}
\hline Name & Solar System Name & $\begin{array}{c}\text { Semimajor Axis } \\
\text { (au })\end{array}$ & $\begin{array}{c}\text { Eccentricity } \\
\ldots\end{array}$ & $\begin{array}{c}\text { Inclination } \\
\left({ }^{\circ}\right)\end{array}$ & $\begin{array}{c}\text { Tisserand Invariant } \\
\ldots\end{array}$ \\
\hline Comet A & 2P / Encke & 2.212 & 0.848 & 11.8 & 3.026 \\
Comet B & 9P / Tempel 1 & 3.138 & 0.514 & 10.5 & 2.969 \\
Comet C & 31P / Schwassmann-Wachmann 2 & 4.249 & 0.194 & 4.5 & 2.992 \\
\hline
\end{tabular}

Table 3. Outcome for the Solar System Clone Simulations

\begin{tabular}{|c|c|c|c|c|c|}
\hline Name & Solar System Name & Item & $\mathrm{Ve} 2 \mathrm{Sa}$ & Ve2Ju & $55 \mathrm{Cnc}$ \\
\hline Comet A & 2P / Encke & $t_{\mathrm{esc}}$ & $82 \mathrm{kyr}$ & $88 \mathrm{kyr}$ & $21 \mathrm{kyr}$ \\
\hline Comet A & $2 \mathrm{P} /$ Encke & $N_{\mathrm{esc}}$ & 2 & 1 & 97 \\
\hline Comet B & 9P / Tempel 1 & $t_{\mathrm{esc}}$ & $55 \mathrm{kyr}$ & $53 \mathrm{kyr}$ & $25 \mathrm{kyr}$ \\
\hline Comet B & 9P / Tempel 1 & $N_{\mathrm{esc}}$ & 42 & 48 & 58 \\
\hline Comet $\mathrm{C}$ & 31P / Schwassmann-Wachmann 2 & $t_{\mathrm{esc}}$ & $40 \mathrm{kyr}$ & $41 \mathrm{kyr}$ & 8 kyr \\
\hline Comet $\mathrm{C}$ & 31P / Schwassmann-Wachmann 2 & $N_{\mathrm{esc}}$ & 43 & 54 & 98 \\
\hline
\end{tabular}

Note: Mean escape times $t_{\mathrm{esc}}$ and number of escapers $N_{\mathrm{esc}}$ out of 100 comet clones.

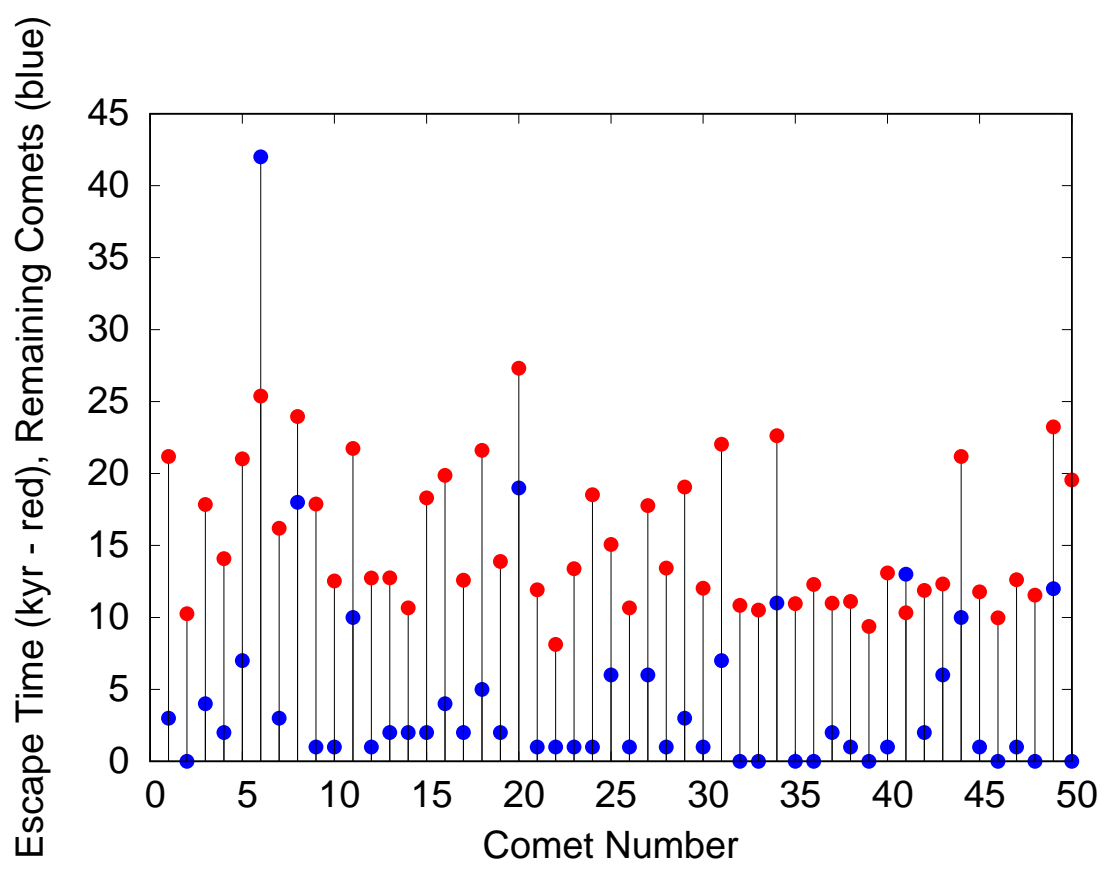

Figure 1. Information on comets no. 1 - 50. Special cases: 1 comet A, 6 comet B, 22 comet C

system presumably after close planetary encounters. Only a few of the comet clones remain in the system toward the end of the integration. One of the comets was pushed to an orbit beyond 55 Cnc-d at approximately 7.9 au where it remained stable with a relatively high eccentricity. In Table 5 the orbital elements of the remaining clones for comet $\mathrm{A}$ are given. One can see that remaining clone no. 4 almost retains its initial eccentricity; however, its inclination is now quite large. It was pushed to an outer orbit at the beginning of the integration and stayed there relatively unperturbed until the integration was stopped. Except for no. 6, all the other comet clones gained higher inclinations during the simulation in compared to their initial values.

Comparing the number of collisions to the number of comets escaping the system yields the following results: About $96 \%$ of all comet clones are ejected from the system after the set integration time of $100 \mathrm{kyr}$. Most of these comets escape after a close encounter with one of the planets. Taking a closer look on the encounters reveals that $78 \%$ of the comet clones pass within a tenth of the planet's Hill 

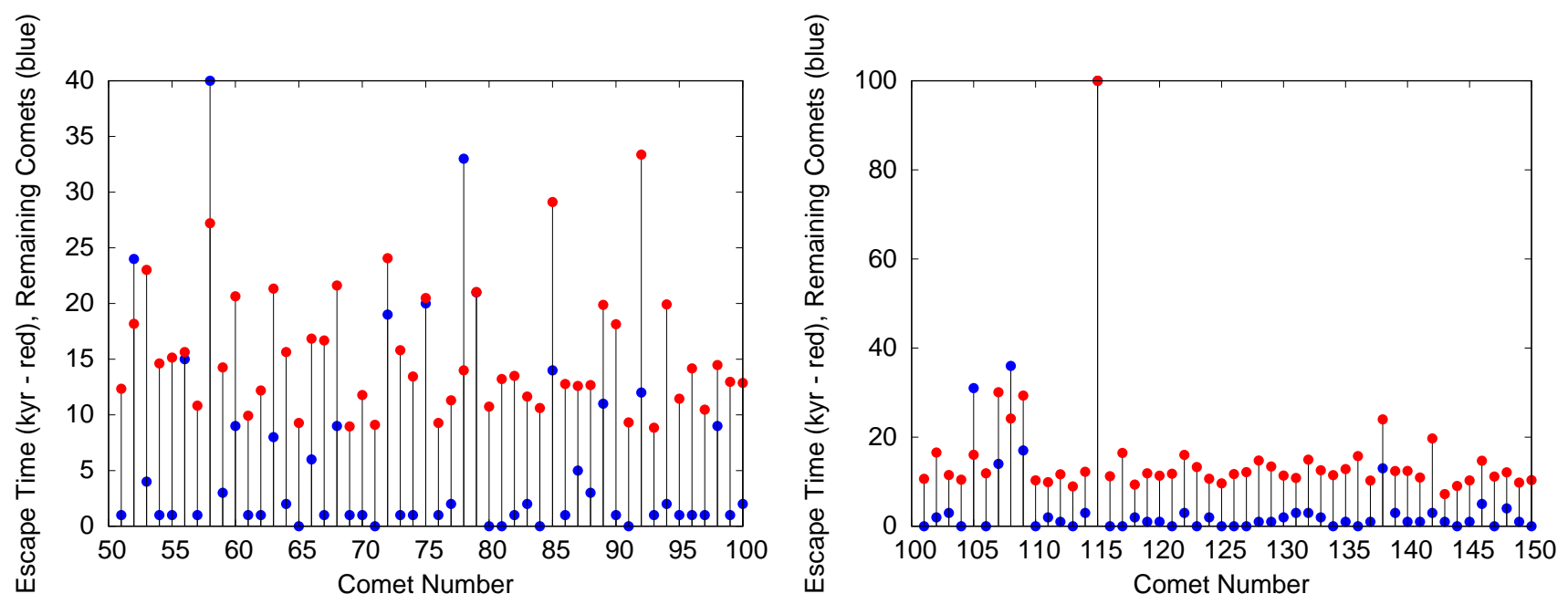

Figure 2. Information on comets no. $51-100$ and no. $101-150$.
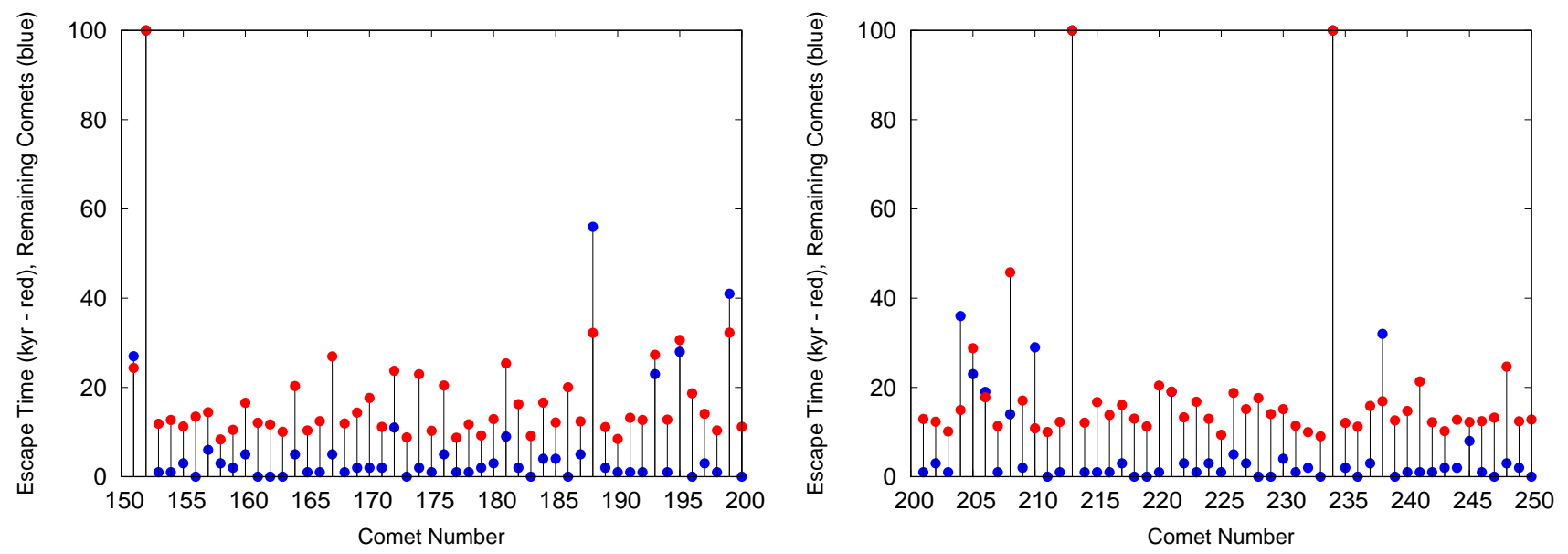

Figure 3. Information on comets no. $151-200$ and no. $201-250$.
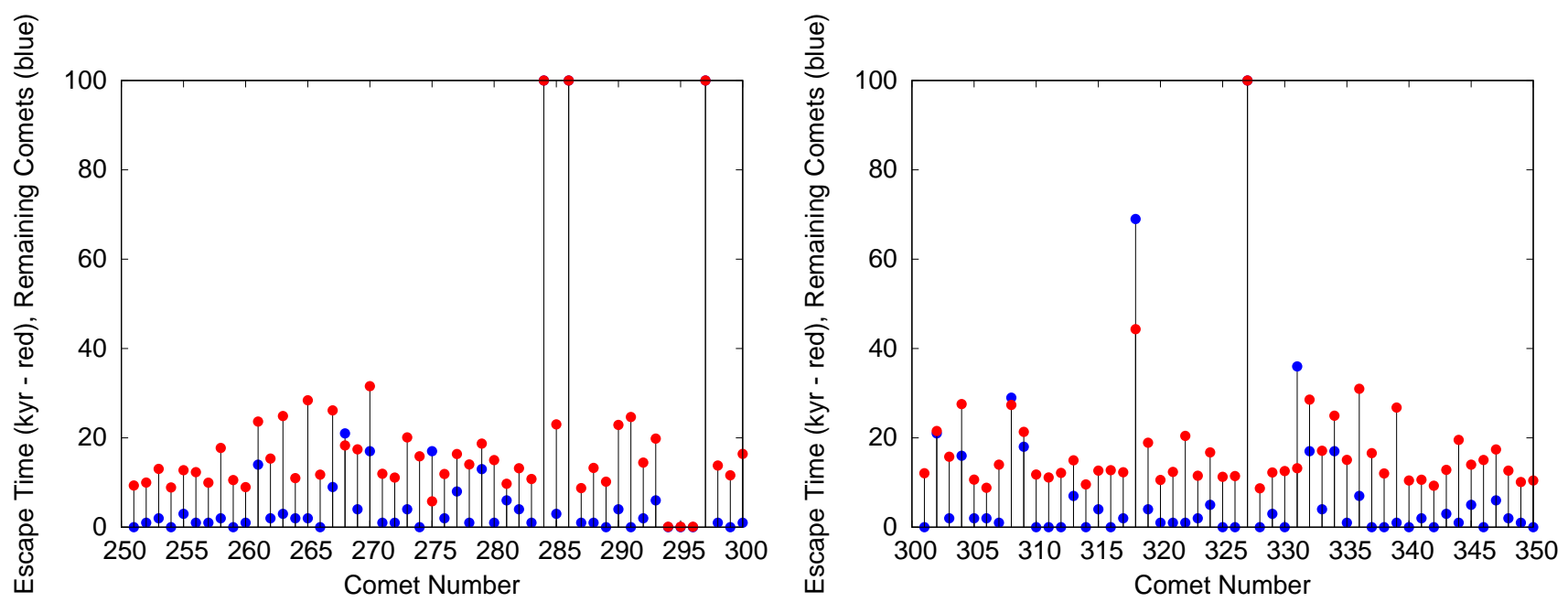

Figure 4. Information on comets no. $251-300$ and no. $301-350$. 

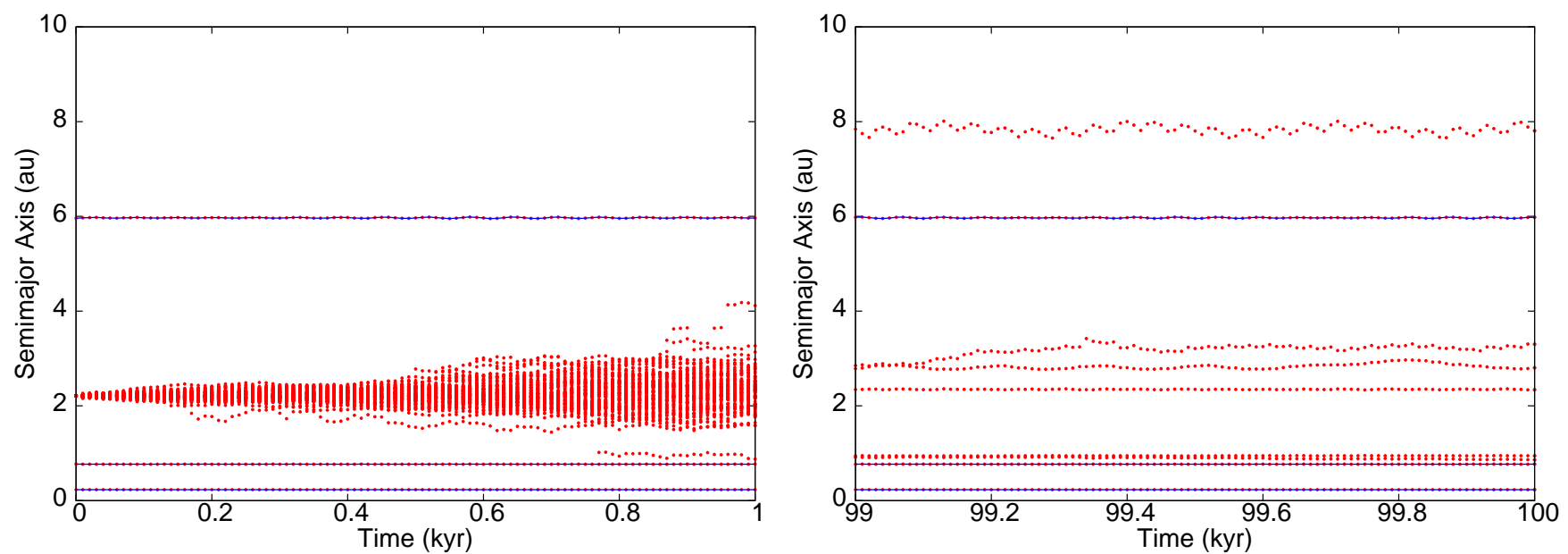

Figure 5. Temporal evolution of the clones of comet A (2P / Encke). Left panel: evolution of the semimajor axes up to 1 kyr; right panel: evolution of the semimajor axes from $99 \mathrm{kyr}$ to $100 \mathrm{kyr}$. Note that up to $1 \mathrm{kyr}$ only a few clones remain captured close to the star. The blue lines denote the planetary distances, whereas the red points represent the clones.

Table 4. Stable JFC Analogs in 55 Cnc

176P / LINEAR
238P / Read
259P / Garradd
311P / PanSTARRS
313P / Gibbs
324P / La Sagra
358P / PanSTARRS
$\mathrm{P} / 2015$ X6 (PanSTARRS)
$\mathrm{P} / 2016$ G1 (PanSTARRS)
$\mathrm{P} / 2019$ A4 (PanSTARRS)
$\mathrm{P} / 2019$ A7 (PanSTARRS)

Table 5. Information on the Remaining Clones of Comet A

\begin{tabular}{lccc}
\hline $\begin{array}{l}\text { Number } \\
\ldots\end{array}$ & $\begin{array}{c}\text { Semimajor Axis } \\
(\mathrm{au})\end{array}$ & $\begin{array}{c}\text { Eccentricity } \\
\ldots\end{array}$ & $\begin{array}{c}\text { Inclination } \\
\left({ }^{\circ}\right)\end{array}$ \\
\hline 1 & 2.8035504 & 0.7199375 & 62.0223301 \\
2 & 3.2998323 & 0.6339047 & 32.4305304 \\
3 & 0.9461796 & 0.5210701 & 28.4985011 \\
4 & 18.4898757 & 0.8552442 & 41.0065245 \\
5 & 7.8064482 & 0.5519021 & 62.1844130 \\
6 & 2.3411522 & 0.5798915 & 8.6652167 \\
7 & 0.8607623 & 0.6551015 & 19.0532704 \\
\hline
\end{tabular}

radius - but still above the presumed planetary surface; note that $91 \%$ of those have a close encounter with planet 55 Cnc-d. The majority of those are ejected from the system as they gain additional energy as a result of the close planetary encounter. Moreover, a small fraction of the comets approach the planet as close as 1/100 Hill radius (approximately the distance of the planet's surface); those comets can thus be assumed hitting the planet (collision). This happens for $\approx 1 \%$ of the total number of comet clones; again, $94 \%$ of the collisions occur with planet 55 Cnc-d.

\subsubsection{The Comet 9P / Tempel 1}

To further our comparison between the Solar System and 55 Cnc we also focused on the comet 9P / Tempel 1, referred to as comet $\mathrm{B}$, while assuming the following initial conditions: $a=3.14$ au adopted as $a=3.59$ au because of the larger distance of planet 55 Cnc-d $(a=5.74 \mathrm{au})$ compared to Jupiter $(a=5.2 \mathrm{au})$. Furthermore, we assume $e=0.514$ and $i=10.5^{\circ}$ (both unaltered). Subsequently, we compared the results of our simulations of up to $100 \mathrm{kyr}$ regarding the orbital elements and the Tisserand parameter for the 100 comet clones. The comparison for the Tisserand parameters is given in Figure 6 and 7. The Earth-mass planet has been included as before. Note that the 100 clones of 9P / Tempel 1 have been created for the purpose of our numerical simulations through varying the initial mean anomalies $M A$ while maintaining the same values for all other initial parameters (see Sect. 3.1).

Figure 6 shows the Tisserand parameter for each comet clone after an integration time of $100 \mathrm{kyr}$. The left panel depicts the system 55 Cnc using the initial values of table 1 without the Earth-mass planet included. The right panel depicts the same kind of simulation but with the fictitious Earth-mass planet included. It is evident that the red dots - corresponding to the Tisserand parameter of the 9P / Tempel 1 clones in the Solar System - show no significant deviations from the value $\mathrm{T}=3$. However, in the system of 55 Cnc some of the comet clones experience orbital changes resulting in significantly different numbers. For better visibility we increased the Tisserand parameters for each clone (purple points) by a factor of 10 .

Figure 7 depicts the semimajor axes of the various comet clones. Again, the left panel depicts the results without the fictitious Earth-mass planet. Here some comet clones within 55 Cnc experience significant orbit changes pushing them out to orbits with high values for the semimajor axis. In contrast, regarding the Solar System comet clones with initial mean anomalies between $170^{\circ}$ and $270^{\circ}$ remain within their initial orbits while maintaining low values for semimajor axis.

We also re-investigated the orbit of 9P / Tempel 1 in 

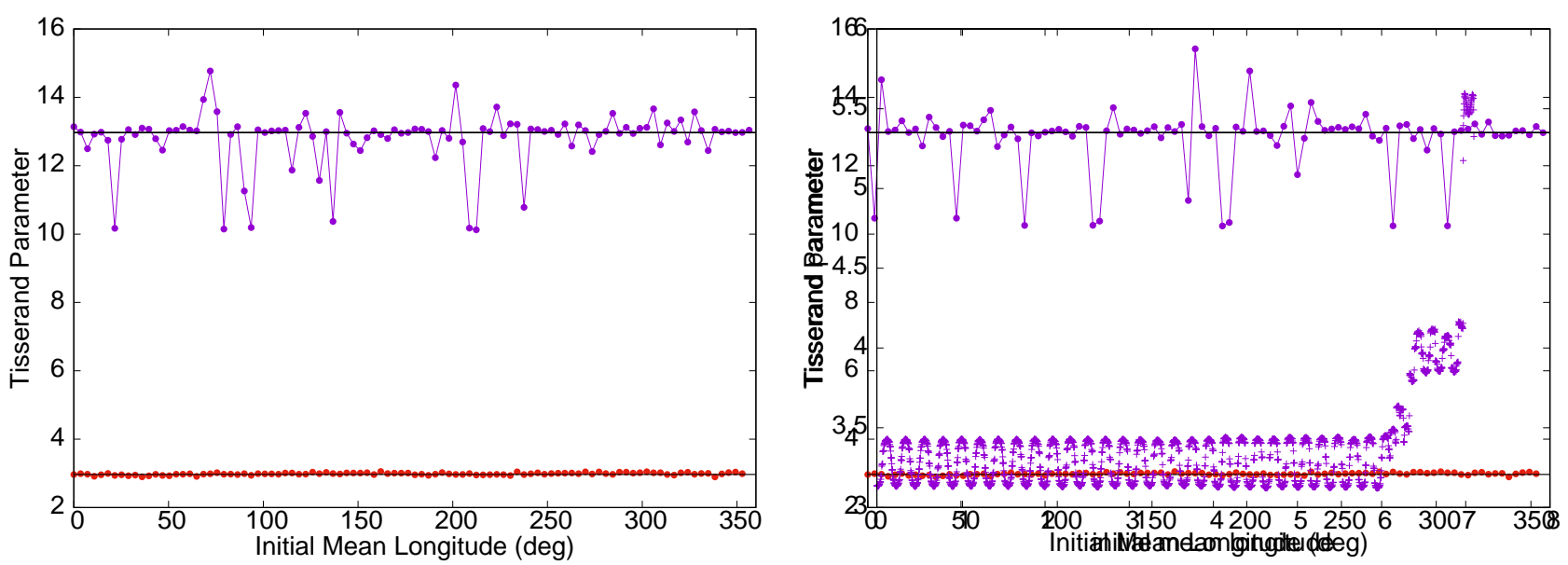

Figure 6. Study of the Tisserand parameter for $9 \mathrm{P} /$ Tempel 1, represented by 100 clones per model. Here we show the results for clones placed into the 55 Cnc system (purple points, values plus 10 for better visibility) and the Solar System (red points). The left panel considers the common system of 55 Cnc (see Table 1), whereas regarding the right panel a fictitious planet Earth at 1 au has been added.
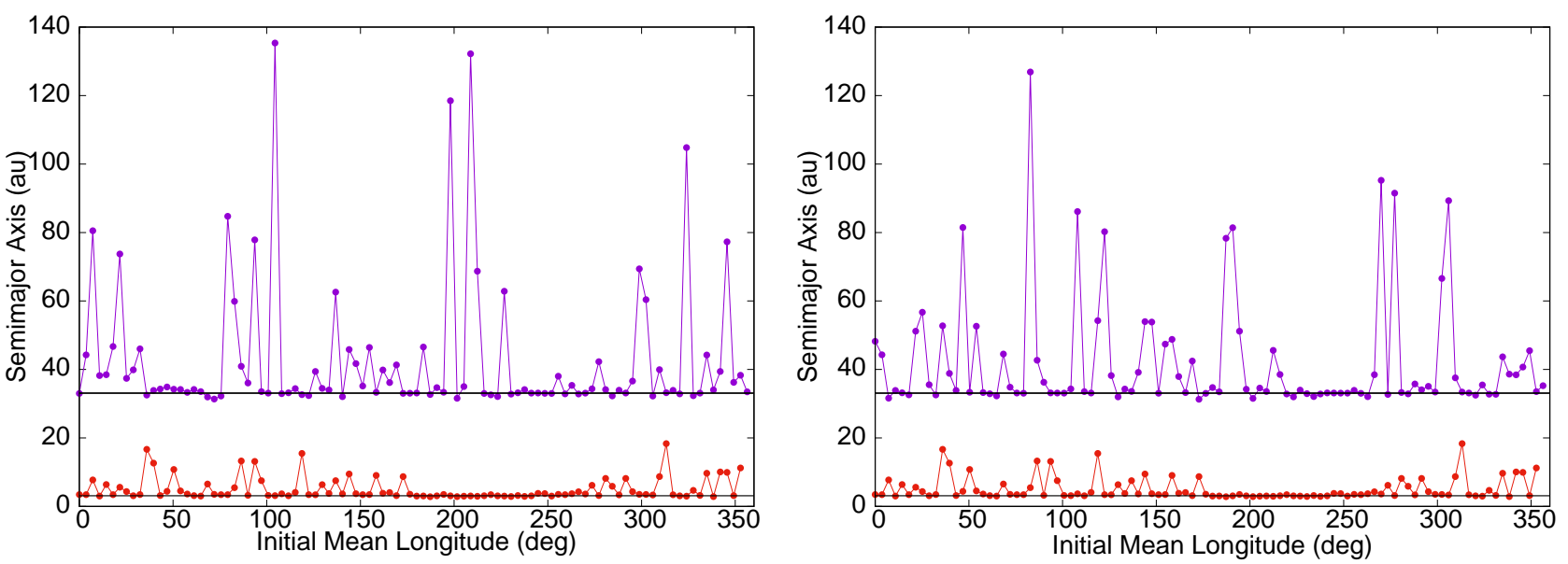

Figure 7. Study of the semimajor axis for 9P / Tempel 1, represented by 100 clones per model. Here we show the results for clones placed into the $55 \mathrm{Cnc}$ system (purple points, values plus 30 for better visibility) and the Solar System (red points). The left panel considers the common system of 55 Cnc (see Table 1), whereas regarding the right panel a fictitious planet Earth at 1 au has been added.

the Solar System and found that some comet clones experience close encounters with the Earth, see Figure 8. The yellow line corresponds to the distance of the Moon from the gravitational centre of the Earth (0). Figure 9 shows the orbit in the in the $x-y$ plane of $9 \mathrm{P} /$ Tempel 1 in our Solar System. Evidently, close encounters with Earth or Mars are expected to happen during the comet's orbital evolution, but no encounters with Venus are possible.

The time evolution of the perihelion and semimajor axis for 9P / Tempel 1 are shown in Figure 9. This comet starts at an orbit with a perihelion distance close to Mars's orbit (red line in Figure 9). After about $7 \mathrm{kyr}$, changes in the semimajor axis occur leading to a decrease in the pericentre distance from the Sun. The comet crosses Earth's orbit at 1 au after about 8 kyr. However, a close approach to Earth happens only after about $9.5 \mathrm{kyr}$.

\section{CONCLUSIONS AND FURTHER RESEARCH}

The aim of this study was to explore the behaviour of fictitious JFCs in the 55 Cnc star-planet system. 55 Cancri (or, technically, 55 Cnc A) bears some resemblance to the Sun, although various differences exist. It has a mass of $0.96 M_{\odot}$ (Ligi et al. 2016), which is lower than the solar mass. Furthermore, its effective temperature and luminosity are below the solar values as well. Moreover, 55 Cnc is known to host five planets. All of them have masses significantly larger than Earth. In addition, following observational constraints, there is a gap without planets between $\sim 0.8$ au and $\sim 5.7$ au, offering the principle possibility of habitable terrestrial planets, including the prospect of long-term orbital stability.

What is the main result of our investigation pertaining to the orbital integration of $\sim 60,000$ fictitious JFCs in 55 Cnc implemented through the study of comet clones? In order to answer this question, each comet clone has been integrated for 100 kyr. Comet clones have been considered 


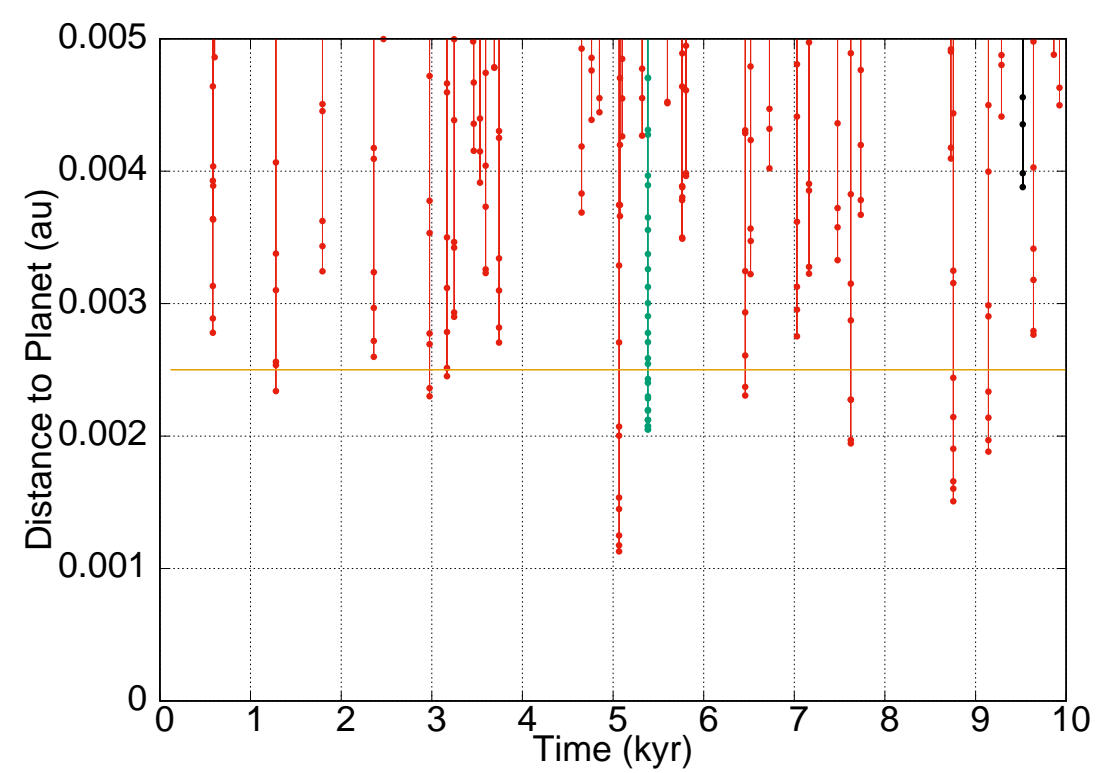

Figure 8. Close encounters of the clones of 9P / Tempel 1 with the Earth in our Solar System. The $y$-axis shows the distance of the object from the gravitational centre of the body undergoing a close encounter. The yellow line at 0.0025 denotes the distance of the Moon from the gravitational centre of the Earth. The red lines depict the distances for a clone from Mars, whereas the green line shows a close encounter of a clone with Jupiter. The black line shows the approach of a clone towards Earth.
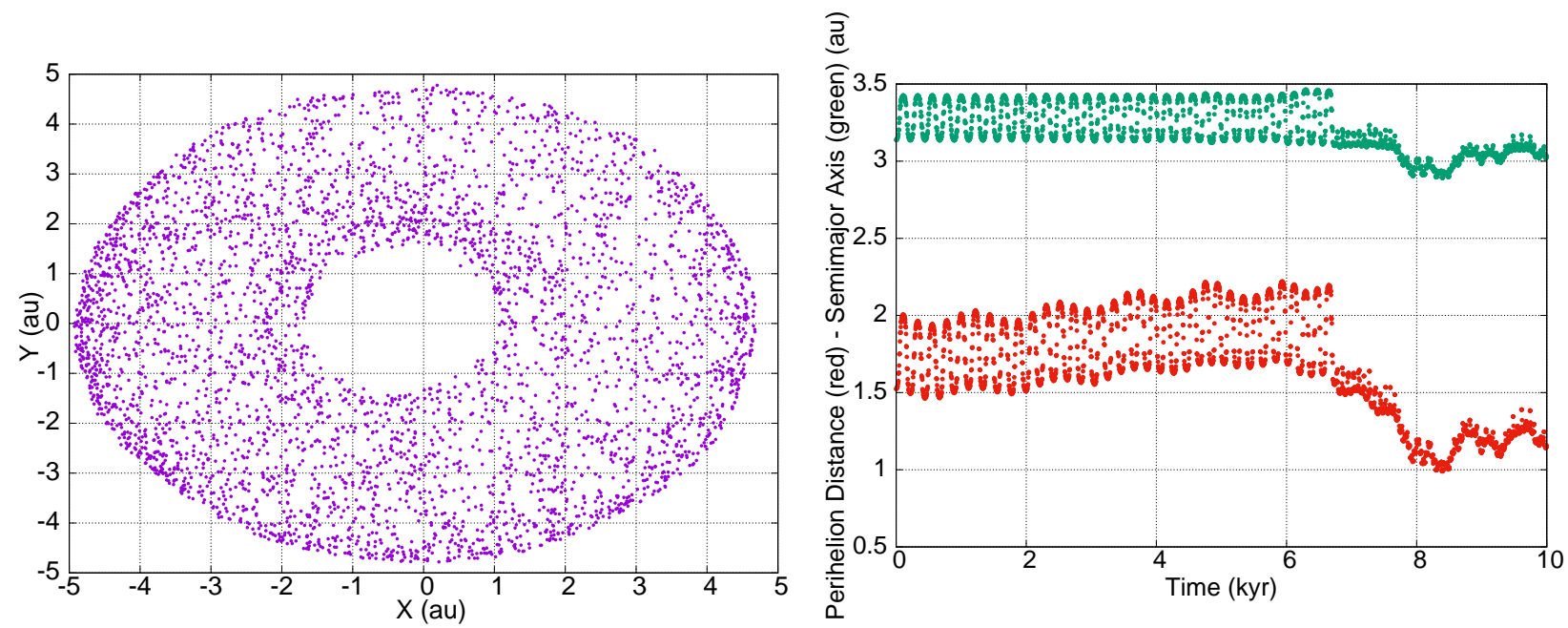

Figure 9. Left: Cometary orbits regarding 9P / Tempel 1 in our Solar System with the $x$ and $y$-axis in au and the Sun situated at the centre at $(0,0)$. Right: The red line corresponds to the perihelion distance of the comet clone, whereas the green line corresponds to its semimajor axis. It is found that it crosses Mars's orbit $(1.5 \mathrm{au})$ and at about 8 kyr it even reaches Earth's orbit $(1 \mathrm{au})$.

for $2 \mathrm{P}$ / Encke, 9P / Tempel 1, and 31P / SchwassmannWachmann 2.

In conclusion, we found that the duration of stability for JFC analogs in $55 \mathrm{Cnc}$ is much less compared to the corresponding comets of the Solar System. This outcome occurs because of the profound differences in the structure of the planetary system of 55 Cnc relative to the Solar System. Between 55 Cnc-f and 55 Cnc-d, located at 0.781 au and $5.74 \mathrm{au}$, respectively, the comets in $55 \mathrm{Cnc}$ do not undergo close planetary encounters akin to Solar System comets with respect to Earth and Mars. The reason is that the planets of the 55 Cnc system possess a significantly higher mass compared to the Solar System planets; consequently, the comets in the 55 Cnc system exhibit a lower level of orbital stability. On the other hand, an increased number of encounters and collisions would be expected between comets and terrestrial planets regarding the $0.8 / 5.7$ au gap.

The region between $0.8 \mathrm{au}$ and $5.7 \mathrm{au}$ allows terrestrial planets to exhibit long-term orbital stability and potential habitability; in fact, this region also encompasses 55 Cnc's habitable zone (e.g., Raymond et al. 2008; Satyal \& Cuntz 2019). Therefore, comets entering this domain are expected to be relevant for the proliferation of water and prebiotic matter to be provided through comet-planet collisions. Hence, there is a need for targeted future observations, especially through the usage of large telescopes, as, e.g., the James Webb Space Telescope ${ }^{5}$. The overall structure of

5 The James Webb Space Telescope (JWST) is a large, spacebased observatory optimized for infrared wavelengths for observ- 
55 Cnc's planetary system, including the possible impact of comets, shall be considered a prodigious inspiration for future theoretical work.

\section{ACKNOWLEDGEMENTS}

This research is supported by the Austrian Science Fund (FWF) through grant S11603-N16 (R. D. and B. L.). Moreover, M. C. acknowledges support by the University of Texas at Arlington. The computational results presented have been achieved in part using the Vienna Scientific Cluster (VSC).

\section{REFERENCES}

Beust H., Vidal-Madjar A., Ferlet R., Lagrange-Henri A. M., 1990, A\&A, 236, 202

Cuntz M., Guinan E. F., 2016, ApJ, 827, 79

Cuntz M., Loibnegger B., Dvorak R., 2018, AJ, 156, 290

Dawson R. I., Fabrycky D. C., 2008, ApJ, 722, 937

Duquennoy A., Mayor M., 1991, A\&A, 248, 485

Dvorak R., Loibnegger B., Cuntz M., 2020, in Prialnik D., Barucci M. A., Young L. A., eds, The Trans-Neptunian Solar System, Elsevier Academic Press, Amsterdam, p. 331

Eiroa C., Rebollido I., Montesinos B., et al., 2016, A\&A, 594, L1

Emel'yanenko V. V., Asher D. J., Bailey M. E., 2013, EM\&P, 110, 105

Endl M., Robertson P., Cochran W. D., et al., 2012, ApJ, 759, 19

Fischer D. A., Valenti J., 2005, ApJ, 622, 1102

Fischer D. A., Marcy G. W., Butler R. P., et al., 2008, ApJ, 675, 790

Fouchard M., Rickman H., Froeschlé Ch., Valsecchi G. B., 2017, Icarus, 292, 218

Fouchard M., Higuchi A., Ito T., Maquet L., 2018, A\&A, 620, A 45

Gaidos E., Mann A. W., Lépine S., et al., 2014, MNRAS, 443, 2561

Gonzalez G., 1998, A\&A, 334, 221

Hanslmeier A., Dvorak R., 1984, A\&A, 132, 203

Kiefer F., Lecavelier des Etangs A., Boissier J., et al., 2014, Nature, 514, 462

Levison H. F., Duncan M. J., 1997, Icarus, 127, 13

Ligi R., Creevey O., Mourard D., et al., 2016, A\&A, 586, A94

Lingam M., Loeb A., 2018, Int. J. Astrobiol., 17, 116

Lingam M., Loeb A., 2019, Int. J. Astrobiol., 18, 527

Loibnegger B., Dvorak R., Cuntz M., 2017, AJ, 153, 203

Mamajek E. E., Hillenbrand L. A., 2008, ApJ, 687, 1264

Newton E. R., Irwin J., Charbonneau D., Berlind P., Calkins M. L., Mink J., 2017, ApJ, 834, 85

Rappaport S., Vanderburg A., Jacobs T., et al., 2018, MNRAS, 474, 1453

Raymond S. N., Izidoro A., 2017, Icarus, 297, 134

Raymond S. N., Barnes R., Gorelick N., 2008, ApJ, 689, 478

Rickman H., Gabryszewski R., Wajer P., et al., 2017, A\&A, 598, A110

Rivera E., Haghighipour N., 2007, MNRAS, 374, 599

Satyal S., Cuntz M., 2019, PASJ, 71, 53

Schwarz R., Bazsó Á., Georgakarakos N., et al., 2018, MNRAS, 480,3595

Schwieterman E. W., Reinhard C. T., Olson S. L., Harman C. E., Lyons T. W., 2019, ApJ, 878, 19

Smith A. W., Lissauer J. J., 2009, Icarus, 201, 381

ing terrestrial planets but with many also many other scientific goals. It is scheduled to be launched in 2021 .
Stern A., 1987, Icarus, 69, 185

von Bloh W., Cuntz M., Franck S., Bounama C., 2003, Astrobiol., 3,681

von Braun K., Boyajian T. S., ten Brummelaar T. A., et al., 2011, ApJ, 740, 49

Winn J. N., Matthews J. M., Dawson R. I., et al., 2011, ApJ, 737, L18

This paper has been typeset from a $\mathrm{TE}_{\mathrm{E}} \mathrm{X} / \mathrm{LATEX}$ file prepared by the author. 